\title{
Thinking Reverse Robin Hood Syndrome in the Emergency Room: Case of a Male with Vertigo
}

\author{
(1) Sadaf Sheikh ${ }^{1}$, (1) Umair Javed ${ }^{2}$, (1) Muhammad Akbar Baig 3 \\ 1Department of Emergency Medicine, Sultan Qaboos University Hospital, Muscat, Oman \\ 2Department of Internal Medicine, Aga Khan University Hospital, Karachi, Pakistan \\ ${ }^{3}$ Department of Emergency Medicine, Aga Khan University Hospital, Karachi, Pakistan
}

\begin{abstract}
Reversed Robin Hood syndrome (RRHS) is described as the steal of arterial blood flow from ischemic to non-ischemic parts of the brain. It is one of the causes of early deterioration in patients with ischemic stroke. The case presented here led us to think about the relationship between RRHS and stroke risk. Flow steal with arterial occlusions is a well-known phenomenon. This phenomenon is named so due to its similarity with the phrase "rob the poor to feed the rich." However, more studies are needed to evaluate the concept of blood flow steal in real-time. A possible mechanism is the vasodilation of non-ischemic areas that steal the blood flow from the ischemic areas.
\end{abstract}

Keywords: Reversed Robin Hood syndrome, stroke, vertigo

\section{Introduction}

Early neurological deterioration (END) with alternatively known as early stroke progression or stroke in evolution is seen in $5-40 \%$ of patients with acute ischemic stroke. It is associated with increased risk of morbidity and mortality (1). It is mainly dealt with the hemodynamic impairment in particularly the vulnerable penumbral areas led to poor cerebral perfusion. The role of trans-cranial Doppler (TCD) to see the hemodynamics in real time is needed in limited cases. Alexandrov et al. (2) described the term RRHS related to decrease in middle cerebral artery flow velocities due to fatigued vasomotor reactivity distal to arterial occlusion led to flow steal directed towards non affected areas (1). Perfusion studies are standard of care as per stroke guidelines (2). Due to the rarity of this pathology, it is important for emergency physicians to have an awareness regarding the condition and its management.

\section{Case Report}

A previously healthy 47 years old Filipino male, shipyard worker by profession with past history of hypertension presented in emergency department with history of intermittent spells of black outs and vertigo for the past one month. It occurred initially while he was at work and denied presence of any neurological symptoms at rest. Later it occurred occasionally over the period of one month with no association with position and usually it lasted for a few seconds. There were no fever, chest pain, headache, tinnitus, excessive exposure to sunlight and nausea or vomiting. There were no associated auditory disturbances or tinnitus or any recent history of flu. Patient denied any previous or recent history of heart disease. Personal history revealed no history of smoking however the patient admitted occasional consumption of alcohol.

Initial physical examination revealed a middle-aged male patient lying comfortably in bed. Vitals were: blood pressure: 144/100 $\mathrm{mmHg}$, pulse: $74 / \mathrm{min}$, respiratory rate: 20 per minute and $\mathrm{O}_{2}$ sats $100 \%$ on room air. On general physical examination, patient looked flushed with no jaundice, clubbing, koilonychia, palpable cervical, axillary and inguinal lymph node, or edema.

Modified Rankin score was 0. Postural drop was negative. Central nervous system examination revealed Glasgow Coma Scale of $15 / 15$ and pupils were equally reactive to light bilaterally.

Cite this article as: Sheikh S, Javed U, Baig MA. Thinking Reverse Robin Hood Syndrome in the Emergency Room: Case of a Male with Vertigo. Eurasian J Emerg Med. 2020;19(4):236-8.

(C) Copyright 2020 by the Emergency Medicine Physicians' Association of Turkey

Eurasian Journal of Emergency Medicine published by Galenos Publishing House. 
Visual acuity was normal and no gross visual field abnormality was noticed on examination. Normal power and tone in upper limb and lower limb with bilaterally down-going plantars were noticed. Reflexes were unremarkable. Cerebellar signs were negative with no pronator drift or nystagmus. Initial lab work up showed in Table 1. Urine toxicology screen, lipid panel and HbA1C were unremarkable. Troponin times 2, echocardiogram and ultrasound neck were unremarkable.

Magnetic resonance imaging (MRI) of brain with angiogram revealed complete occlusion of left internal carotid artery (ICA) with loss of flow void in the right internal jugular vein with possibility of thrombus (Figure 1). However, it revealed no acute

\begin{tabular}{|l|l|}
\hline \multicolumn{2}{|l|}{ Table 1. Laboratory parameters } \\
\hline Hemoglobin & 25 \\
\hline Hematocrit & 77 \\
\hline Mean corpuscular volume & 96.6 \\
\hline White blood cells & 7.6 \\
\hline Neutrophils & 69.3 \\
\hline Lymphocytes & 22.2 \\
\hline Platelets & 205 \\
\hline Blood urea nitrogen & 15 \\
\hline Creatinine & 0.7 \\
\hline Sodium & 133 \\
\hline Potassium & 3.7 \\
\hline Chloride & 105 \\
\hline Bicarbonate & 20.5 \\
\hline Calcium & 9.4 \\
\hline Magnesium & 1.9 \\
\hline
\end{tabular}

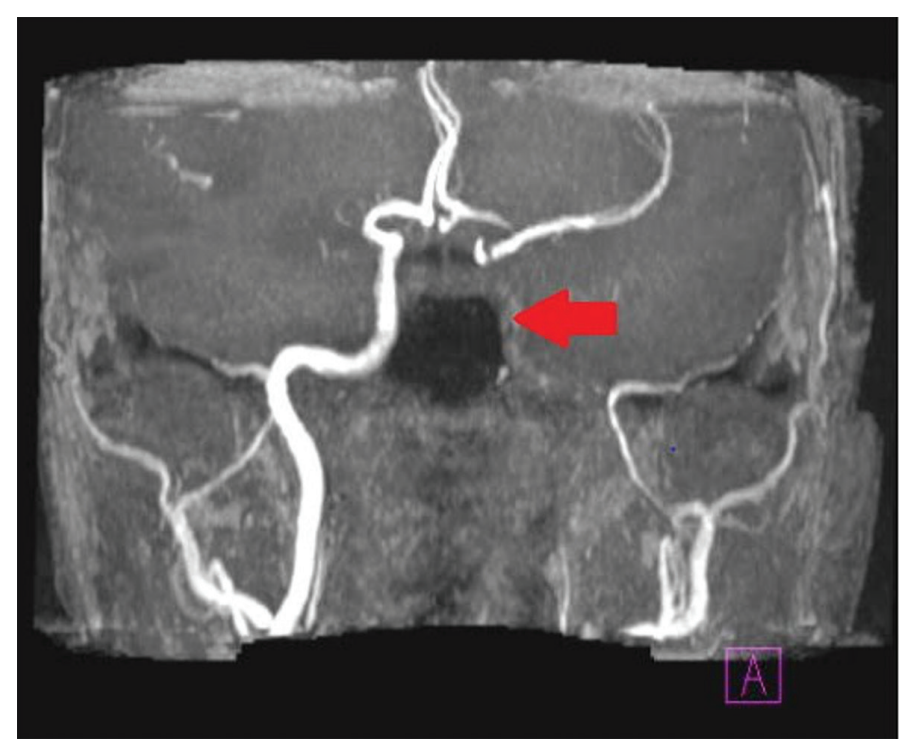

Figure 1. Occluded left carotid artery as seen in magnetic resonance imaging infarction, intracranial hemorrhage or mass effect. Additionally, it showed hyper-intense foci on T2/FLAIR in periventricular deep white matter suggestive of micro-vascular ischemic changes. Magnetic resonance venography was negative for cerebral venous sinus thrombosis and internal jugular vein thrombosis. He was prescribed enoxaparin $60 \mathrm{mg}$ twice daily.

Due to the personal issues, patient left without medical advice from our facility. He was counselled for symptoms recurrence with vertigo, aphasia, visual disturbances and stroke in particular. He was also advised for TCD and neurological follow up as soon as possible. It was advised to consider options like transluminal angioplasty with stenting of the intracranial ICA or to be kept on dual anti-platelet therapy. Later, he went lost to follow up.

\section{Discussion}

RRHS demonstrates exhausted vasodilatory response when blood vessels distal to proximal occlusion failed to further dilate in response to stimuli. There is a flow steal from ischemic to nonischemic areas (1). RRHS coexist also in the absence of a collateral mechanism hence recurrence of impaired perfusion and stroke. Definite treatment is endovascular stenting with symptoms regression in few week-time. Fewer studies discussed the role of medical treatment versus stenting suggesting trans-luminal stenting effective and safe (1). Medical treatment includes calcium channel blockers, intra-arterial milrinone, anticoagulation and blood pressure management (2-4). Intra-arterial vasodilators are showing promise as a mainstay treatment option. Enhanced external counter pulsation therapy is the newest form of the therapy (4).

TCD and serial NIH stroke scale were used in ICA occlusion for RRHS. It is detected as transient vasodilatory stimuli induced velocity reductions in affected arteries. In case of confirmed steal, RRHS many suggest target population in stroke patients (2).

In conlusion, we describe a case of patient with vertigo and MRI brain showing complete occlusion of ICA and subsequent RRHS which might cause recurrent episodes of focal neurological deterioration in the same territories. Further validation of this relation is needed in association with TCD. However, more studies are needed to evaluate the concept of blood flow steal in real time.

\section{Ethics}

Informed Consent: The authors certify that they have obtained verbal patient consent forms. The patient understands that his name and initial will not be published and due efforts will be made to conceal their identity.

Peer-review: Externally peer-reviewed. 


\section{Authorship Contributions}

Surgical and Medical Practices: S.S., U.J., M.A.B., Concept: S.S., U.J., M.A.B., Design: S.S., U.J., M.A.B., Data Collection or Processing: S.S., U.J., M.A.B., Analysis or Interpretation:S.S., U.J., M.A.B., Literature Search: S.S., U.J., M.A.B., Writing: S.S., U.J., M.A.B.,

Conflict of Interest: No conflict of interest was declared by the authors.

Financial Disclosure: The authors declared that this study received no financial support.

\section{References}

1. Geng HH, Wang Q, Li B, Cui BB, Jin YP, Fu RL, et al. Early neurological deterioration during the acute phase as a predictor of long-term outcome after first-ever ischemic stroke. Medicine. 2017;96:e9068.

2. Alexandrov AV, Nguyen HT, Rubiera M, Alexandrov AW, Zhao L, Heliopoulos I, et al. Prevalence and risk factors associated with reversed Robin Hood syndrome in acute ischemic stroke. Stroke. 2009;40:2738-42.

3. Alexandrov AV, Sharma VK, Lao AY, Tsivgoulis G, Malkoff MD, Alexandrov AW. Reversed Robin Hood syndrome in acute ischemic stroke patients. Stroke. 2007;38:3045-8.

4. Zhao M, Guan L, Wang Y. The association of autonomic nervous system function with ischemic stroke, and treatment strategies. Front Neurol. 2019;10:1411. 